\title{
GPER-1 acts as a tumor suppressor in ovarian cancer
}

\author{
Tanja Ignatov ${ }^{1 \dagger}$, Saskia Modl ${ }^{1,2+}$, Maike Thulig ${ }^{1}$, Christine Weißenborn ${ }^{1,2}$, Oliver Treeck ${ }^{3}$, Olaf Ortmann³, \\ AC Zenclussen ${ }^{2}$, Serban Dan Costa ${ }^{1}$, Thomas Kalinski ${ }^{4}$ and Atanas Ignatov ${ }^{1,3^{*}}$
}

\begin{abstract}
Background: It is known that the new membrane-bound estrogen receptor GPER-1 acts suppressive in breast cancer cells and its expression decreases during disease progression. This study was conducted to evaluate the GPER-1 expression in ovarian cancer and its correlation with progression. Its function was tested in vitro in ovarian cancer cells.

Patients and methods: GPER-1 expression was analyzed by immunohistochemistry in 35 benign ovarian tumors, 35 tumors of low-malignant potential and in 124 ovarian cancers. GPER-1 expression was correlated to the prospectively evaluated disease-free survival of ovarian cancer patients. We also tested GPER-1 expression in ovarian cancer cells and the effect of GPER-1 stimulation on cell growth.

Results: GPER-1 expression was significantly lower in ovarian cancer tissue than in benign and low-malignant ovarian tumors. GPER-1 expression was observed in $83.1 \%$ of malignant tumors and was higher in early stage cancers and tumors with high histological differentiation. GPER-1 expression was associated with favourable clinical outcome. The difference in 2-year disease-free survival by GPER-1 expression was significant, 28.6\% for GPER-1 negative and 59.2\% for GPER-1 positive cases $(p=0.002)$. GPER-1 expression was observed in SKOV-3 and OVCAR-3 ovarian cancer cell lines. G-1, a selective GPER-1 agonist, suppressed proliferation of the two cell types via inhibition of cell cycle progression in G2/M phase and stimulation of caspase-dependent apoptosis. The blockade in G2/M phase was associated with increased expression of cyclin B1 and Cdc2 and phosphorylation of histone 3.
\end{abstract}

Conclusion: GPER-1 emerges as a new tumor suppressor with unsuspected therapeutic potential for ovarian cancer.

Keywords: GPR30, GPER-1, Ovarian cancer

\section{Introduction}

Ovarian cancer is a common neoplasm in Western countries and more than $70 \%$ of all diagnoses are in advanced stage $[1,2]$. The heterogeneity of ovarian cancer forces the researcher to find new strategies and targets to individualize the therapy of this common disease [3]. Recently the G-protein-coupled receptor 30 was claimed to be a new membrane-bound G-protein-coupled estrogen receptor-1 (GPER-1) involved in the rapid

\footnotetext{
* Correspondence: atanas.ignatov@gmail.com

${ }^{\dagger}$ Equal contributors

'Department of Obstetrics and Gynecology, Otto-von-Guericke University,

G.Hauptmann Str. 35, 39108, Magdeburg, Germany

${ }^{3}$ Department of Obstetrics and Gynecology, University Medical Center

Regensburg, Regensburg, Germany

Full list of author information is available at the end of the article
}

nongenomic effects of estrogen in normal and cancer tissue [4]. It belongs to the family of G-protein-coupled receptors (GPCRs) and acts independently of estrogen receptor (ER) $\alpha$ and ER $\beta$ [4]. GPER-1 stimulation is associated with increased cAMP production assuming a coupling of GPER-1 to Gos [5]. Moreover, GPER-1 activation leads to activation and release of heparin-bound growth factor (HB-EGF), which in turn activates the epidermal growth factor receptor (EGFR) followed by phosphorylation of MAPK [6]. However, the physiological and pathophysiological function of GPER-1 remains incompletely discovered.

Despite the fact that GPER-1 can mediate the proliferative effects of estrogen in many estrogen-related cancers, we and others have demonstrated that GPER-1 can

\section{Biomed Central}


inhibit cell proliferation in different cell systems [7-11]. Moreover, we have recently found that GPER-1 downregulation in breast cancer tissue is associated with poor clinical outcome [12].

The role of GPER-1 in ovarian carcinogenesis is only particularly and very controversial studied. Smith and co-workers have shown in 89 ovarian cancer patients that GPER-1 expression is associated with poor survival [13]. In contrast, Kolkova and co-workers have not found any correlation between GPER-1 expression and survival of 152 patients with ovarian cancer [14]. A third research group has found that GPER-1 expression predicts lower survival of 150 ovarian cancer patients only by co-expression with epidermal growth factor receptor (EGFR) [15]. In vitro studies provide also controversial data regarding GPER-1 effect on cell growth [16], adding confusion to the role of GPER-1 in ovarian cancer.

This study was undertaken to put more clearance in the role of GPER-1 in ovarian tumor biology. We investigated GPER-1 protein expression in benign and malignant ovarian tumors. GPER-1 expression was correlated to clinicopathological characteristics and clinical outcome of ovarian cancer patients, which was assessed prospectively. In addition, the effect of GPER-1 stimulation in ovarian cancer cells was assayed via its specific agonist G-1.

\section{Patients and methods}

\section{Patients and tissue samples}

The data of 35 patients with benign ovarian tumors and 35 patients with ovarian tumors of low malignant potential (LMP), who had been admitted to the Department of Obstetrics and Gynecology, Otto-von-Guericke University, Magdeburg, Germany from 1999 to 2011, were selected by retrospective analysis. Additionally, tissue from 124 ovarian cancer patients was obtained at the operation in the Department of Obstetrics and Gynecology, University Clinic of Magdeburg, Germany in the period between 2005 and 2010. The study was approved by the Research and Ethical Committee of Otto-von-Guericke University, Magdeburg, Germany. The expression analysis of GPER-1 in ovarian cancer tissue was designed as a prospective monocentre cohort study. The primary outcome of the study was the correlation of GPER-1 expression and the 2-year disease-free survival (DFS) of ovarian cancer patients. Outcome was measured as DFS, according to the International Union Against Cancer (UICC) criteria [17]. Secondary outcome was the correlation of GPER-1 expression in ovarian cancer tissue and clinicopathological characteristics. The minimal follow-up period was 24 months. The inclusion criteria were diagnosis of ovarian cancer, no previous treatment with chemotherapy and no history of other carcinomas. Exclusion criteria included a previous history of adjuvant anti-hormonal or cytostatic therapy, incomplete adjuvant chemotherapy, no sufficient material for detection of GPER-1 expression and incomplete 24 months follow-up data. One hundred thirty seven eligible patients were enrolled in the study. Thirteen patients were secondary excluded because of lost of followup (7 patients), incomplete chemotherapy (3 patients) and detection of second cancer during the follow-up time (3 patients). All ovarian cancer patients underwent adjuvant platinum-based chemotherapy.

The median age at the time of primary diagnosis was 62 years (range 12-87 years) in the group of patients with benign ovarian tumors, 52 years (range $21-81$ years) in the group of patients with LMP tumors and 64 years (range 20-86 years) in the group of patients with ovarian cancer.

\section{Immunohistochemistry}

GPER-1 expression was analyzed as previously described [18]. Briefly, sections of formalin-fixed and paraffinembedded ovarian tumors, including benign ovarian cyst, tumors of low malignant potential (LMP) and malignant ovarian cancer specimens were investigated. The immunostaining was performed with affinity-purified rabbit antibody against GPER-1 (SP4677P; Acris antibodies, Herford, Germany) diluted 1:500. The slides were counterstained with hematoxylin and cover slipped after being embedded in mounting medium. The specificity of the antibody has been already proven [18]. Moreover, we assayed GPER-1 expression on 15 representative tissue samples of ovarian carcinoma with a second antibody against GPER-1 (sc48524-R, Santa Cruz, Heidelberg, Germany diluted 1:500). The obtained expression pattern with the two antibodies was very similar and confirms again their specificity (data not shown).

GPER-1 expression was classified as already described $[18,19]$, according to the following grading system: staining extensity was categorized as 0 (no positive cells), 1 (<10\% positive cells), 2 (10-50\% positive cells), or 3 ( $>50 \%$ positive cells), and staining intensity was categorized as 0 (negative), 1 (weak), 2 (moderate), or 3 (strong). The individual categories were multiplied to give a total immunohistochemical score (IHC). IHC score ranged between 0 and 9 . GPER-1-positive expression was defined for tumors that showed an $\mathrm{IHC} \geq 1$. Representative example of GPER-1 expression is shown in Figure 1.

\section{Cell culture and treatment}

Ovarian cancer cell lines SKOV-3 and OVCAR-3 were obtained from Cell Lines Services (Germany) and routinely cultured in DMEM/F12 (PAN Biotech) supplemented with $10 \% \mathrm{FBS}, 100 \mathrm{U} / \mathrm{ml}$ penicillin and $100 \mu \mathrm{g} / \mu \mathrm{l}$ streptomycin at 


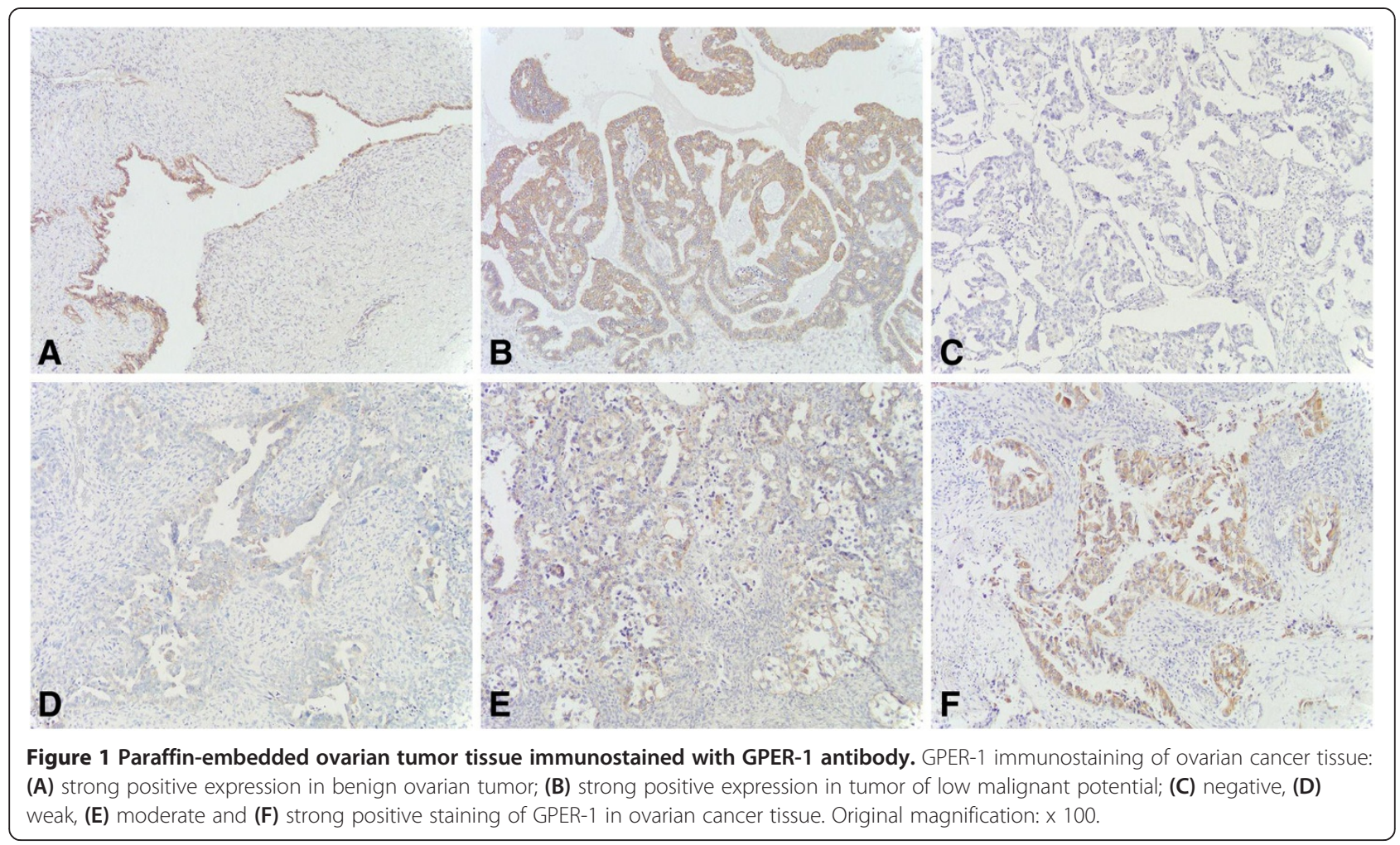

$37^{\circ} \mathrm{C}$ in a humidified $5 \% \mathrm{CO}_{2}$ atmosphere. The cells were treated as indicated in the figure legends.

\section{Western blotting}

Western blotting procedures were performed as previously described [18]. Briefly, the blots were incubated overnight at $4^{\circ} \mathrm{C}$ with antibodies against GPER-1 (sc48524-R, Santa Cruz, Heidelberg, Germany, diluted 1:500), caspase-3 (ab13847, Abcam, Cambridge, UK, diluted 1:1000), cyclin B1(\#4138, Cell signalling, Frankfurt am Main, Germany, diluted 1:1000), Cdc2 (\#9112, Cell signalling, Frankfurt am Main, Germany, diluted 1:2000) phosphoH3(Ser10) (\#9701, Cell signalling, Frankfurt am Main, Germany, diluted 1:2000) and for $2 \mathrm{~h}$ at room temperature with antibody against $\beta$-actin (A5441, Sigma Aldrich, Hamburg, Germany diluted 1:10000). Peroxidase-conjugated anti-rabbit and anti-mouse antibodies (Thermo Scientific, Waltham, MA, USA), diluted 1:2000 and 1:5000, were used as secondary antibodies ( $2 \mathrm{~h}$ at room temperature), respectively.

\section{Cell cycle analysis and apoptosis assay}

Cell lines were treated with $1 \mu \mathrm{M}$ G-1 or vehicle control for 48-72 h. Cell cycle distribution was analysed by propidium iodide (PI) staining using flow cytometry. Apoptosis was determined by using the FITC Annexin V Apoptosis Detection Kit (BD, Heidelberg, Germany).

\section{MTT-viability assay}

MTT-viability assay was performed as already described [20]. Briefly, 2000 cells per well were seeded and cultured in 96-well plate in growth medium. After $24 \mathrm{~h}$ the cells were stimulated with indicated concentrations of G-1, G-15 or DMSO as control for 5 days. This was followed by a $3 \mathrm{~h}$ treatment with MTT (3-[4,5dimethylthiazol-2-yl]-2,5-diphenyltetrazolium bromide) in the dark at $37^{\circ} \mathrm{C}$. After removing the supernatants and incubation in $150 \mu \mathrm{l}$ lysis buffer (isopropanol containing $4 \mathrm{mM} \mathrm{HCl}$ and $0,1 \% \mathrm{NP}-40$ ) cell viability was determined by measuring the absorbance at $570 \mathrm{~nm}$ (Synergy HT, BioTek, Germany).

\section{Statistical analysis}

The statistical calculations were performed using SPSS Version 20.0 (SPSS, Chicago, IL, USA). An association between GPER-1 expression and the tumor variables was evaluated using the $\mathrm{x}^{2}$ test or Fisher's exact test. DFS analysis took into account those who died of ovarian cancer-specific death or had a recurrence of disease as a primary event. Patients who died of other causes were censored. Survival was calculated using the Kaplan-Meier method. The equality of survival curves was tested by the log rank test. Univariate Cox proportional hazard regression analysis was used to identify significant prognostic factors. The prognostic significance was confirmed by multivariate analysis. The statistical 
analyses were two sided and p-values of $<0.05$ were considered statistically significant. The comparison of GPER-1expression between benign tumors, LMP tumors and ovarian cancers was analyzed using nonparametric paired test, and was performed with the Wilcoxon signed rank sum test.

\section{Results}

GPER-1 protein expression in ovarian tumors and correlation with clinical and pathologic features of ovarian cancer

GPER-1 expression was assessed by immunohistochemistry in 35 benign ovarian tumors, 35 ovarian tumors of low malignant potential (LMP) and 124 ovarian cancers. GPER-1 expression was observed in all (100\%) benign ovarian tumors, in 34 of 35 (97.1\%) tumors of LMP and in 103 of 124 (83.1\%) of ovarian cancers. GPER-1 expression was significantly lower in ovarian cancer compared to the benign and tumors of LMP $(\mathrm{p}<0.0001$; Figure 2A). The median IHC-score was 6 for benign and tumors of LMP, whereas ovarian cancer tissue demonstrated a median IHC-score of 2 .

Next, we compared GPER-1 protein expression with the clinicopathological features of ovarian cancer. GPER1 expression was significantly associated with the clinical stage of disease ( $p=0.032$; Table 1$)$. Loss of GPER-1 expression was more frequently observed in advanced ovarian cancer. Moreover, a reduction of GPER-1 expression of approximately $20 \%$ was observed with decrease of histological differentiation (Table 1). However, this correlation did not reach a significant level. There was no correlation between GPER-1 immunostaining and other tumor characteristics (Table 1). All this data suggested a loss of expression of GPER-1 during ovarian tumorigenesis.

\section{GPER-1 expression and clinical outcome of ovarian cancer patients}

During the prospective follow-up time of 24 months we observed 67 recurrences of cancer. There was a significant difference in DFS between GPER-1-positive and GPER-1-negative patients (Table 1). GPER-1 positivity was associated with reduced rate of disease recurrence $(\mathrm{p}=0.008)$.

Univariate analysis revealed that GPER-1 expression was a favorable prognostic factor for DFS (HR, 0.404; 95\% CI, 0.233-0.732; $p=0.003$ ) (Table 2). The 2-year DFS was $28.6 \%$ for GPER-1-negative patients and $59.2 \%$ for GPER-1-positive patients ( $p=0.002$; Figure 2B). After adjustment for patient age, menopausal status, tumor stage, lymph node status, histological grading, multivariate analysis rendered GPER-1 as an independent, favorable prognostic factor in regard to DFS (HR, 0.569; 95\% CI, 0.317-0.991; $\mathrm{p}=0.046$ ) (Table 2). Tumor stage was evaluated as an unfavorable independent prognostic factor (HR, 2.764; 95\% CI, 1.590-3.751; p < 0.0001).

\section{Effect of GPER-1 stimulation on ovarian cancer cell proliferation}

Next we wanted to test the effects of GPER-1 in vitro. First of all, we analyzed the protein expression of GPER-1 in SKOV-3 and OVCAR-3 ovarian cancer cells by western blot (Figure 3A). MCF-7 breast cancer cells were used as positive control. GPER-1 expression was observed in the two ovarian cell lines. However, OVCAR-3 cells demonstrated a 2-fold higher GPER-1

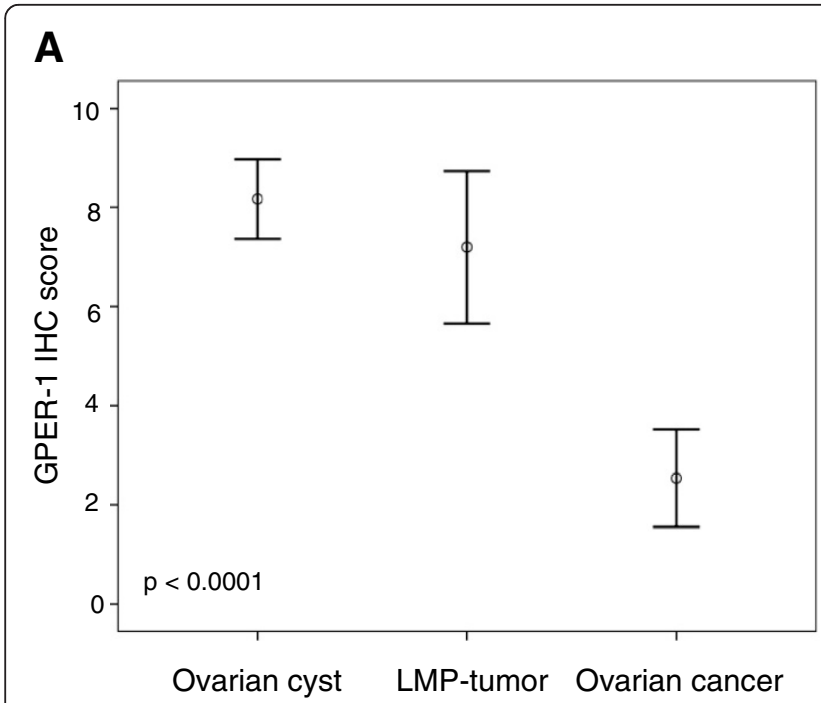

B

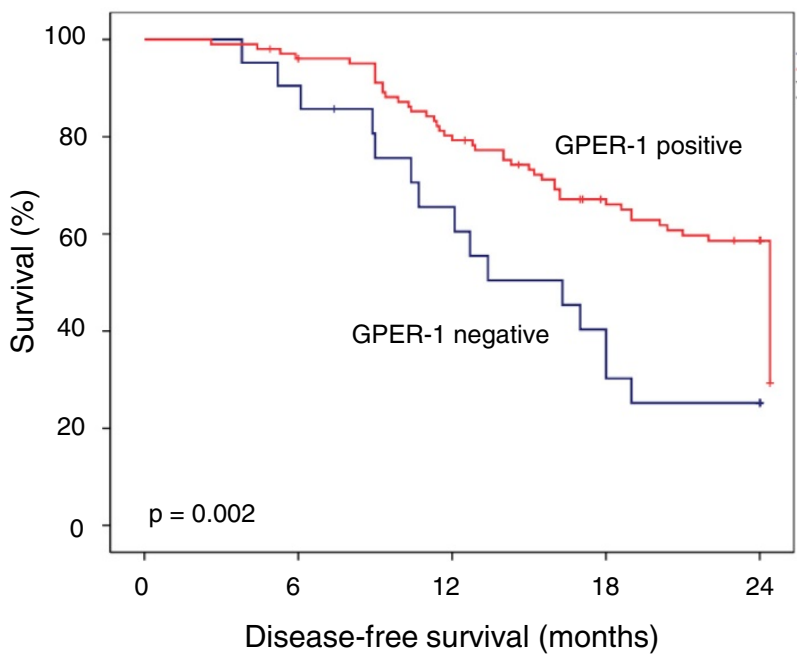

Figure 2 GPER-1 protein expression and clinical outcome. A) Protein expression of GPER-1 in ovarian tumors. B) Disease-free survival of ovarian cancer patients according to GPER-1 expression. The log rank test was used to calculate the p-value. 
Table 1 Characteristics of study cohort

\begin{tabular}{|c|c|c|c|c|}
\hline \multirow[b]{2}{*}{ Characteristic } & \multirow{2}{*}{$\begin{array}{c}\mathrm{N} \text { of } \\
\text { patients }\end{array}$} & \multicolumn{2}{|c|}{ GPER-1 } & \multirow[b]{2}{*}{ p-value } \\
\hline & & $\begin{array}{c}\text { Negative } \\
\mathrm{N}(\%)\end{array}$ & $\begin{array}{c}\text { Positive } \\
\text { N (\%) }\end{array}$ & \\
\hline Total & 124 & $21(16.9)$ & $103(83.1)$ & \\
\hline Age, years (range) & 124 & $65(30-84)$ & $61(20-85)$ & 0.136 \\
\hline \multicolumn{5}{|l|}{ Menopausal status } \\
\hline premenopausal & 16 & $1(6.2)$ & $15(93.8)$ & \\
\hline postmenopausal & 108 & $20(18.5)$ & $88(81.5)$ & 0.304 \\
\hline \multicolumn{5}{|l|}{ FIGO Stage } \\
\hline 1 & 25 & $0(0)$ & $25(100)$ & \\
\hline 2 & 21 & $6(28.6)$ & $15(71.4)$ & \\
\hline 3 & 76 & $14(18.4)$ & $62(81.6)$ & \\
\hline 4 & 2 & $1(50.0)$ & $1(50)$ & 0.032 \\
\hline \multicolumn{5}{|l|}{ Lymph node status } \\
\hline negative & 31 & $5(16.1)$ & $26(83.9)$ & \\
\hline positive & 18 & $3(16.7)$ & 15 (83.3) & 1.000 \\
\hline \multicolumn{5}{|l|}{ Histological grading } \\
\hline 1 & 18 & $0(0)$ & $18(100)$ & \\
\hline 2 & 38 & $7(18.4)$ & $31(81.6)$ & \\
\hline 3 & 68 & 14 (20.6) & $54(79.4)$ & 0.112 \\
\hline \multicolumn{5}{|l|}{ Histology } \\
\hline serous & 81 & $13(16.0)$ & $68(84.0)$ & \\
\hline mucinous & 16 & $3(18.8)$ & $13(81.2)$ & \\
\hline endometrioid & 13 & $2(15.4)$ & $11(84.6)$ & \\
\hline clear cell & 8 & $0(0)$ & $8(100)$ & \\
\hline other & 6 & $3(50)$ & $3(50)$ & 0.171 \\
\hline \multicolumn{5}{|c|}{ Recurrence (in 24 months) } \\
\hline no & 57 & $4(7.0)$ & $53(93.0)$ & \\
\hline yes & 67 & 17 (25.4) & $50(74.6)$ & 0.008 \\
\hline
\end{tabular}

expression than SKOV-3 cells. Then we investigated whether GPER-1 influences the growth of ovarian cancer cells. Both ovarian cell lines were incubated with increasing concentrations of GPER-1 specific agonist G-1. A concentration-dependent inhibition of cell proliferation was observed (Figure $3 \mathrm{~B}$ ). The estimated $\mathrm{IC}_{50}$ value was $3.9 \mu \mathrm{M}$ and $0.8 \mu \mathrm{M}$ for SKOV-3 and OVCAR-3 cells, respectively.

Furthermore, we wanted to exclude a possible cytotoxic effect of high G-1 concentrations. For this purpose we incubated SKOV-3, OVCAR-3 and GPER-1-negative HEK-293 cells with $1 \mu \mathrm{M} \mathrm{G-1}$ for 5 days. As demonstrated in Figure 4, G-1 did not change the proliferation of HEK-293 in comparison to control-treated cells. It suggested that G-1 inhibitory effect in SKOV-3 and OVCAR-3 ovarian cancer cells due to specific activation of GPER-1 and not to cytotoxic properties. To explore the involvement of GPER-1 activation in G-1-induced
Table 2 Uni- and multivariate analysis in regard to DFS

\begin{tabular}{lccc}
\hline Variable & HR & $\mathbf{9 5 \% ~ C l}$ & P-value \\
\hline Univariate analysis & & & \\
GPER-1 expression & 0.404 & $0.233-0.732$ & 0.003 \\
Age & 1.010 & $0.990-1.031$ & 0.322 \\
Menopausal status & 1.676 & $0.715-3.930$ & 0.235 \\
Tumor stage & 3.023 & $1.774-5.150$ & $<0.0001$ \\
Lymph node status & 1.950 & $0.731-5.197$ & 0.182 \\
Histological grading & 2.132 & $1.363-3.335$ & 0.001 \\
Multivariate analysis & & & \\
GPER-1 expression & 0.549 & $0.317-0.991$ & 0.046 \\
Tumor stage & 2.764 & $1.590-3.751$ & $<0.0001$ \\
Histological grading & 1.576 & $0.982-2.531$ & 0.060 \\
\hline
\end{tabular}

cell growth inhibition we extended our experiments using G-15 a recently discovered GPER-1 receptor antagonist [21]. Pre-incubation of SKOV-3 and OVCAR-3 cells with $1 \mu \mathrm{M} \mathrm{G-15}$ was able to block the inhibitory effect of G-1 (Figure 4). As expected in GPER-1-negative HEK-293 cells G-15 pre-incubation was not associated with any changes in cell proliferation (Figure 4). All these data suggested an involvement of GPER-1 in G-1 -induced proliferation effects.

\section{GPER-1 activation by G-1 induced a cell-cycle arrest and} cell apoptosis in SKOV-3 and OVCAR-3 cells

We next tested by flow cytometry analysis whether GPER-1 activation by G-1 results in abrogation of cell cycle progression. Stimulation of ovarian cancer cells with G-1 led to concentration-dependent accumulation of SKOV-3 (Figure 5A) and OVCAR-3 (Figure 5B) cells in apoptotic sub-G1 phase and decreased the cell number in G1 phase. Higher concentrations of G-1 were associated with additional block in $\mathrm{G} 2 / \mathrm{M}$ phase.

To confirm the effect of GPER-1 on cell apoptosis AnnexinV assay was applied. A concentration- and timedependent increase of cell apoptosis was observed in the two cell lines after stimulation with G-1 (Figure 6). In the SKOV-3 cell line we observed a concentrationdependent increase of cell apoptosis by $1 \%, 3 \%$ and $7 \%$ after $48 \mathrm{~h}$ stimulation with $500 \mathrm{nM}, 700 \mathrm{nM}$ and $1 \mu \mathrm{M}$ G-1, respectively (Figure 6A). After $72 \mathrm{~h}$ incubation duplication of G-1-induced cell apoptosis was observed. In OVCAR-3 cells G-1 treatment caused even higher rates of cell apoptosis. After treatment with G-1 for $48 \mathrm{~h}$ in aforementioned concentrations cell apoptosis reached a level of $7 \% 13 \%$, and $28 \%$, respectively (Figure 6B). After $72 \mathrm{~h}$ stimulation the rate of cell apoptosis increased to $13 \%, 30 \%$, and $49 \%$ after incubation with $500 \mathrm{nM}, 700 \mathrm{nM}$ and $1 \mu \mathrm{M}$ G-1 respectively (Figure 6B).

These data suggested that GPER-1 might inhibit ovarian cancer cell proliferation via simultaneous cell cycle 


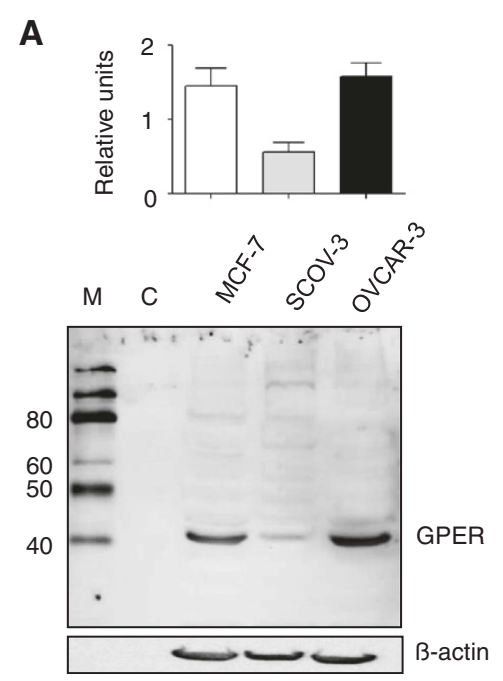

\section{B}

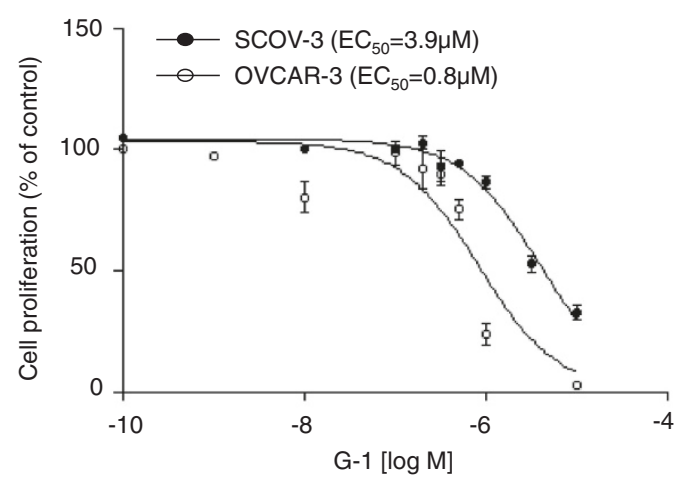

Figure 3 GPER-1 expression and G-1 effect on ovarian cancer cells. A) Representative example of GPER-1 protein expression in MCF-7 breast cancer cells and in SCOV-3 and OVCAR-3 ovarian cancer cells. B-actin was used as a loading control. B) SCOV-3 and OVCAR-3 cells were treated with indicated concentration of G-1 and the cell number was counted using MTT viability assay. Comparison of the dose-response curves yielded $I_{50}$ values of $3.9 \mu \mathrm{M}$ and $0.8 \mu \mathrm{M}$ for SCOV-3 and OVCAR-3, respectively. Each experiment was repeated at least three times. The results are expressed as means $\pm \mathrm{SD}$. M, marker; $\mathrm{C}$, negative control $(\mathrm{H} 2 \mathrm{O})$.

arrest and cell apoptosis. To investigate the G-1-induced cell cycle arrest and apoptosis in more details we examined the expression of different regulatory proteins involved in cell cycle progression and apoptosis. After stimulation of SCOV-3 (Figure 7A) and OVCAR-3 (Figure 7B) cells with $1 \mu \mathrm{M}$ G-1 for indicated time, the expression of cyclin $\mathrm{B} 1$ and $\mathrm{Cdc} 2$, the phosphorylation of histone $\mathrm{H} 3$ and the cleavage of caspase- 3 was measured by immunoblotting. In the two cell lines tested in this study, we found that G-1-induced cell cycle arrest and apoptosis was associated with up-regulation of

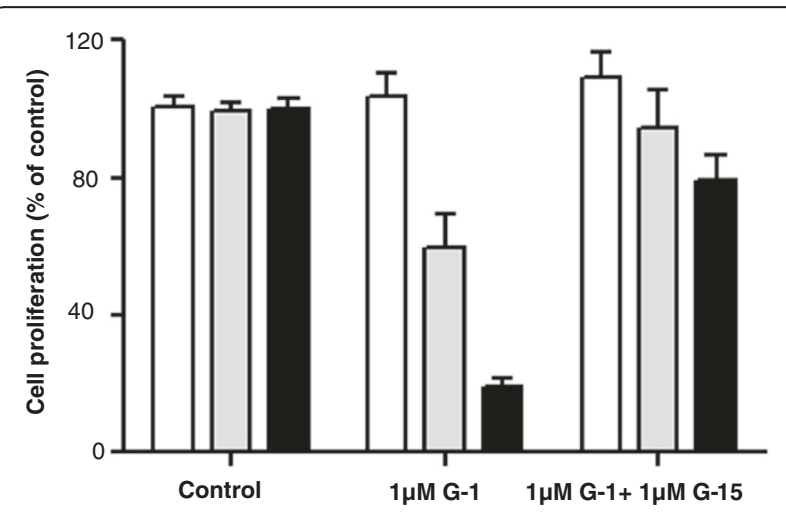

Figure 4 GPER-1 specific antagonist G-15 abolished the G-1-induced inhibitory effect in ovarian cancer cells. GPER-1-positive SCOV-3 and OVCAR-3 ovarian cancer cells and GPER-1-negative HEK-293 cells were incubated for 5 days with 1 MM G-1 with or without pre-treatment with $1 \mu \mathrm{M} \mathrm{G}-15$ for $24 \mathrm{~h}$ and the cell number was counted using MTT viability assay. Each experiment was repeated at least three times. The results are expressed as means \pm SD. cyclin B1 and Cdc2 protein expression. The phosphorylation of histone $\mathrm{H} 3$ was also observed (Figure 7). The levels of cyclin $\mathrm{B} 1$ and $\mathrm{Cdc} 2$ protein expression started to increase at $6 \mathrm{~h}$ after G-1 treatment, reached a peak between 12 and $24 \mathrm{~h}$ followed by rapid decline (data not shown). The phosphorylation of histone H3, indicative for cells in mitotic phase, started to increase at $6 \mathrm{~h}$ after stimulation of the cells with G-1 and peaked at $12 \mathrm{~h}$ (Figure 7).

The caspase- 3 cleavage product as result of its activation was observed in both cell lines after incubation with G-1. The activation of caspase-3 started at $24 \mathrm{~h}$ after GPER-1 activation and peaked around $48 \mathrm{~h}$ in the two cell lines (Figure 7).

\section{Discussion}

This is the first report demonstrating the favourable role of GPER-1 in ovarian cancer patients in regard to disease-free survival (DFS). GPER-1 expression and clinical outcome were investigated in a very homogenous cohort of platinum-treated ovarian cancer patients. GPER-1 expression was a favourable factor regarding 2year DFS in ovarian cancer patients. Moreover, GPER-1 expression decreased from benign to malignant ovarian tumours. In vitro, GPER-1 stimulation was associated with a significant increase of cellular apoptosis and cell cycle arrest in ovarian cancer cells.

To date there are only few reports about GPER-1 and clinical outcome of ovarian cancer patients providing very confusing and controversial results. Smith and coworkers have recently found that GPER-1 expression 

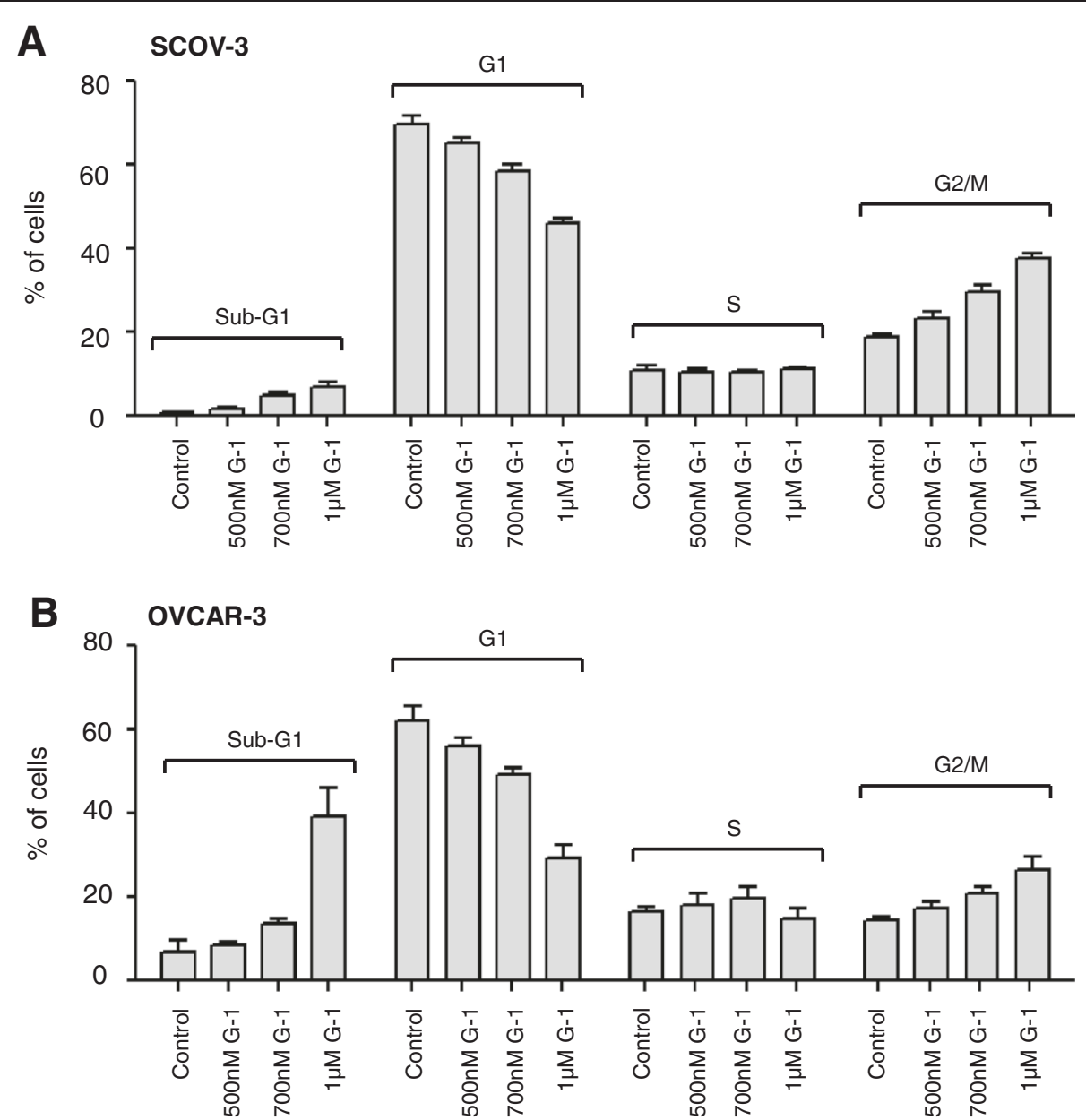

Figure 5 Effects of G-1 on cell cycle progression in ovarian cancer cells. Cell cycle distribution of (A) SCOV-3 and (B) OVCAR-3 cells treated or not with indicated concentrations of G-1 for $48 \mathrm{~h}$ was determined by flow cytometry analysis. Each experiment was repeated at least three times. The results are expressed as means \pm SD.

predicts poor survival of ovarian cancer patients [13], which is in the opposite to our observation. In this report the median overall survival was significantly shorter for patients expressing high levels of GPER-1. It is remarkable, that this correlation was observed only in adverse tumors but not for low grade tumors. Unfortunately, the impact of GPER-1 on patient outcome has not been confirmed via multivariate analysis. In the aforementioned patient collective GPER-1 expression significantly correlated with tumor stage and tumor grading, two known prognostic factors in ovarian cancer patients, which could explain the impact of GPER-1 on survival. In our cohort of patients GPER-1 was independent favourable prognostic factor even after adjustment of other prognostic factors. Another plausible explanation of the controversial results is a difference in population studied. Interestingly, another research group has not found any correlation between GPER-1 expression on mRNA and protein level with clinical outcome of ovarian cancer patients [14]. It is to note that in this cohort of 150 patients GPER-1 was expressed in only one third, whereas in our cohort more than $80 \%$ of the invasive ovarian cancers expressed GPER-1. The difference might be explained by the specificity of the antibodies used, the evaluation criteria of GPER-1 expression or by the fact that Kolkova et al. correlated GPER-1 expression only to overall survival but not to DFS [14]. Similar results were observed in analysis of 152 patients by Fjiwara et al. [15]. In these series GPER1 expression has not shown any correlation with overall survival of ovarian cancer patients. However, the authors stated that EGFR expression in combination with GPER1 predicts lower survival in patients with ovarian cancer. This is in agreement with our recent results 


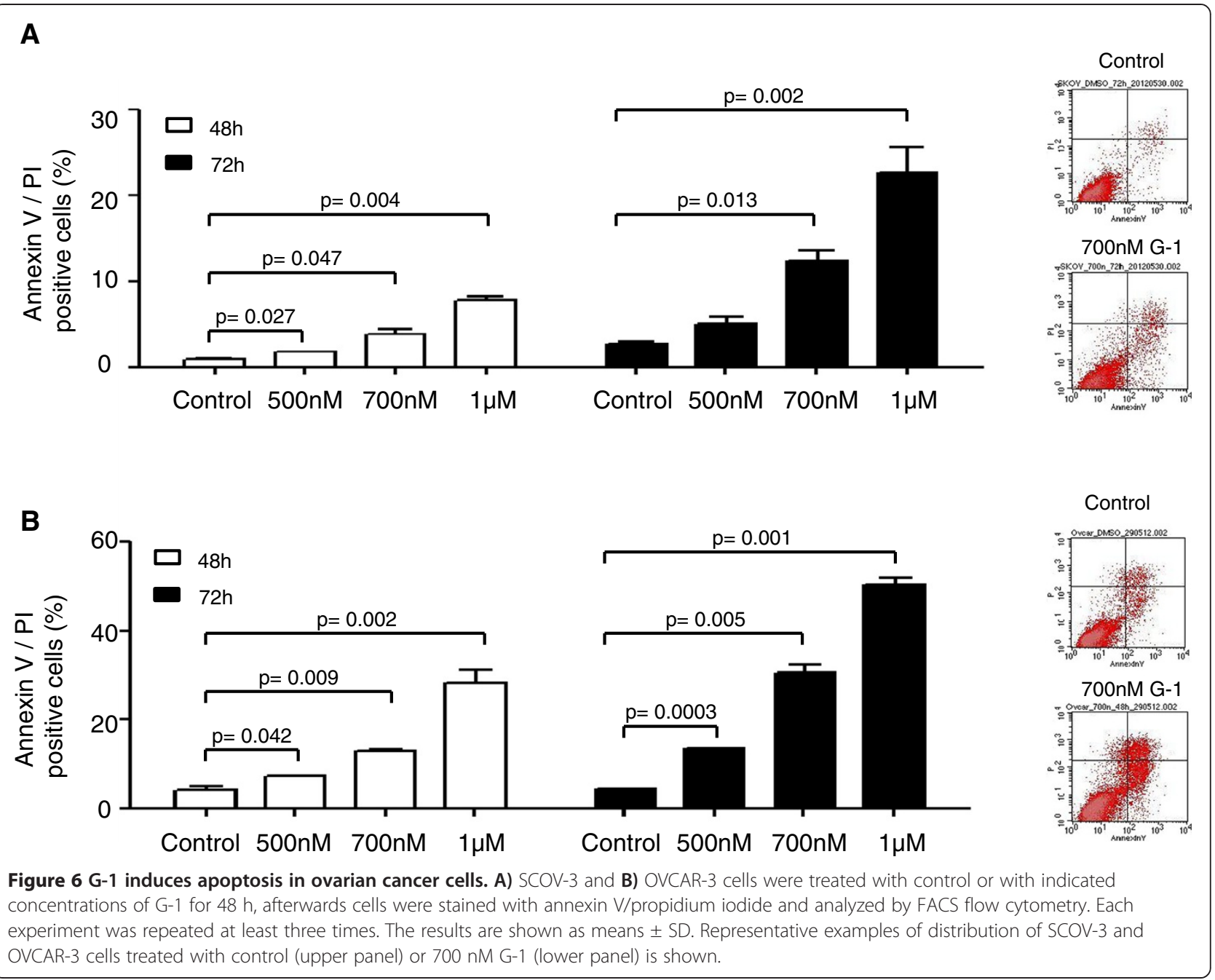

demonstrating that GPER-1 cross-talks with EGFR in breast cancer cell lines [22]. The cross-talk between GPER-1 and EGFR is associated with activation of completely different signalling pathways $[5,6,23]$ and could be responsible for the observed difference in survival rates. In agreement with the results obtained by us in the present study we have recently found that GPER-1 is associated with better patient survival in breast cancer patients [18] and have been recently confirmed for inflammatory breast cancer [24].

In accordance to expressional analyses, in vitro studies revealed controversial results regarding cellular functions of GPER-1. Since establishment of GPER-1 as an estrogen receptor many reports demonstrated its stimulatory effect in breast, ovarian and endometrial cells $[23,25,26]$. Nevertheless, numerous recent investigations clearly demonstrated an inhibitory effect of GPER-1 in various cell systems [7-11,16,27] and are in agreement with our findings. G-1 inhibitory effect in these cells systems has been observed only in high concentrations similar to our results in ovarian cancer cells. The absence of G-1-induced inhibition in GPER-1-negative HEK293 cells exclude a potential cytotoxic effect to the cells.

GPER-1 stimulation inhibited ovarian cell proliferation by inducing cell apoptosis and cell cycle arrest. We observed an accumulation of cyclin $\mathrm{B} 1$ and $\mathrm{Cdc} 2$ regulatory proteins in G-1-treated ovarian cancer cells. This is contradicting the results observed in breast and prostate cancer cells where cyclin B1 and Cdc2 expression were inhibited by G-1 stimulation $[9,10]$. Possible explanation for this discrepancy could be the use of different cell types or the fact that breast cancer cells and prostate cancer cell were blocked in G1 [9] and G2 phase [10], respectively. The suppression of Cyclin $\mathrm{B} 1$ and $\mathrm{Cdc} 2$ is associated with G2 arrest, whereas the up-regulation of the two proteins induced a cell accumulation in the $M$ phase [28]. Thus the G-1-induced activation of Cyclin $\mathrm{B} 1$ and $\mathrm{Cdc} 2$ in ovarian cancer cells is associated with progression in the $G 2$ phase to $M$ phase. It was 


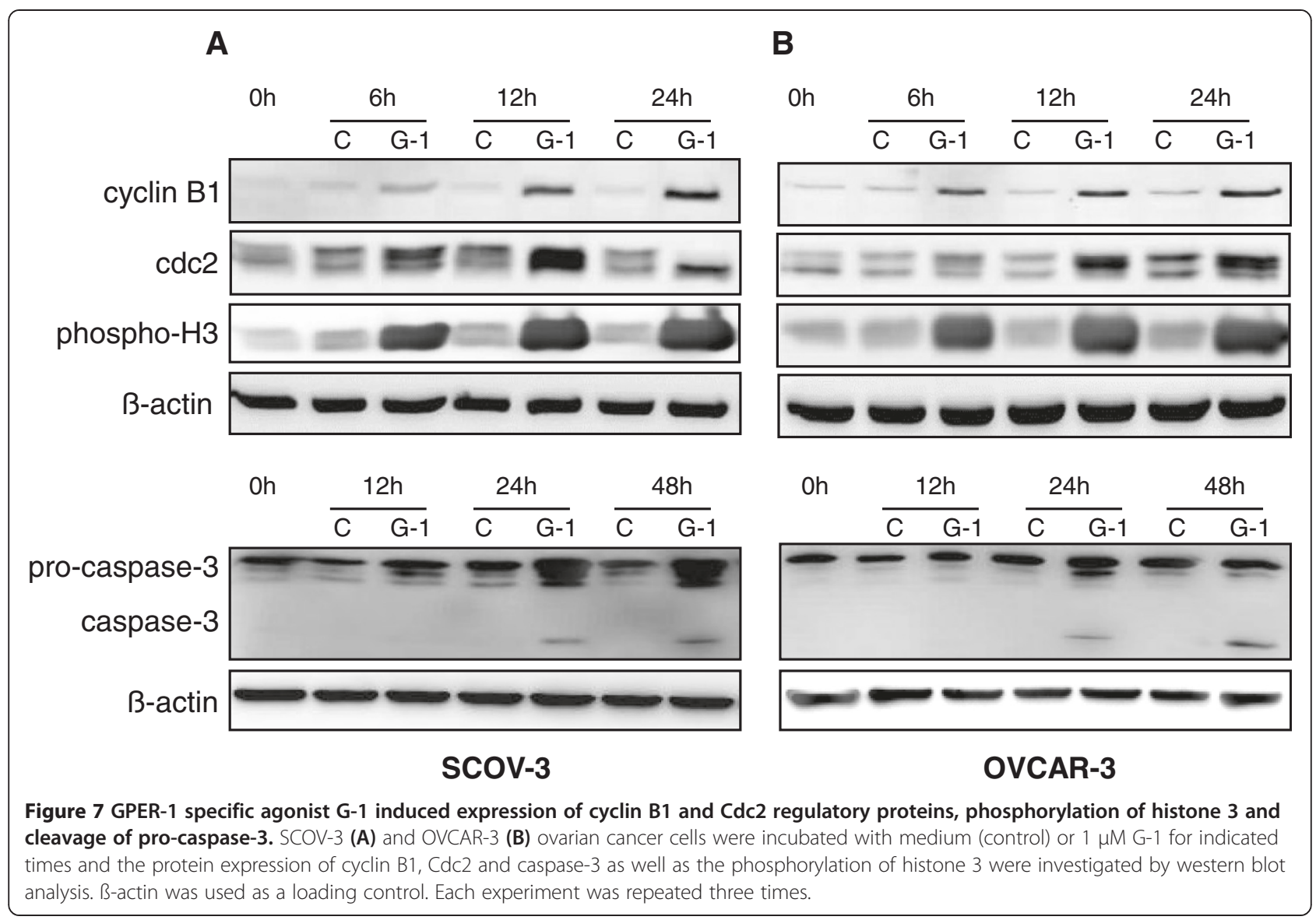

confirmed by the observation that phosphorylation of histone $\mathrm{H} 3$, an important step occurring during G2 to M transition [29], was significantly induced by G-1 activation. The perturbation of mitotic progression by GPER-1 stimulation with consequent increased mitotic duration could trigger caspase-3 cleavage and cell apoptosis. This phenomenon has been already described for taxol and nocodazole, two microtubule inhibitors with anticancer activity $[28,30]$. The inhibitory effect of GPER-1 in ovarian cancer cells has been recently well documented and is in accordance to our results. Henic and co-workers have recently found that GPER-1 stimulation attenuates the invasive properties of ovarian cancer cells [31].

Our results and the review of literature make us to hypothesize that GPER is a potential tumor suppressor. Tumor suppressors are known to be inactivated during cancer progression [32] which was also observed for GPER-1. The decreased expression of GPER-1 benign and malignant ovarian tumors corroborates the presumption that GPER-1 might be a tumor suppressor. This phenomenon was observed by us during the breast cancer tumorigenesis and was confirmed by two other groups $[33,34]$. We have found that GPER-1 is inactivated via promoter methylation in breast cancer tissue and is re-expressed after treatment with demethylating agent 5-Aza (own not published data).

The most important finding of our study is the fact that GPER-1 with its high expression of $80 \%$ in ovarian cancer tissue could be an appropriate target for individualized therapy of ovarian cancer. Our study provides evidence that GPER-1 may be a tumor suppressor gene. First, GPER-1 specific agonist inhibited ovarian cancer cell proliferation by inducing cell apoptosis and partially cell cycle arrest. Second, GPER-1 expression was found to be down-regulated during ovarian cancer tumorigenesis.

\section{Competing interests}

The authors declare that they have no competing interests.

\section{Authors' contributions}

$\mathrm{Tl}$ carried out part of the evaluation of the $\mathrm{IHC}$ analysis, western blot analysis and statistical analysis, participated in the design of the study and analysis of the clinical data and drafted the manuscript; SM carried out the in vitro

assays, MT participated in IHC analysis, CW carried out part of in vitro assays; OT, OO, AZ, SDC participated in study design and analysis of clinical data; TK and $\mathrm{Al}$ carried out part of $\mathrm{IHC}$ analysis, participated in the design of the study and statistical analysis of the clinical data, contributed methodological knowhow and drafted the manuscript. All authors read and approved the final version of the manuscript. All authors read and approved the final manuscript. 


\section{Authors' information}

Thomas Kalinski and Atanas Ignatov contributed equally to this work as senior authors.

\section{Grant support}

This work was supported by grants from the Deutsche Krebshilfe (to A.I.).

\section{Author details}

${ }^{1}$ Department of Obstetrics and Gynecology, Otto-von-Guericke University, G.-Hauptmann Str. 35, 39108, Magdeburg, Germany. ${ }^{2}$ Department of Experimental Obstetrics and Gynecology, University Clinic, Magdeburg, Germany. ${ }^{3}$ Department of Obstetrics and Gynecology, University Medical Center Regensburg, Regensburg, Germany. ${ }^{4}$ Department of Pathology, University Clinic, Magdeburg, Germany.

Received: 9 April 2013 Accepted: 12 July 2013

Published: 13 July 2013

\section{References}

1. Bray F, Loos AH, Tognazzo S, La Vecchia C: Ovarian cancer in Europe: Cross-sectional trends in incidence and mortality in 28 countries, 1953-2000. Int J Cancer 2005, 113:977-990.

2. Jemal A, Siegel R, Xu J, Ward E: Cancer statistics, 2010. CA Cancer J Clin 2010, 60:277-300.

3. Bast RC Jr, Hennessy B, Mills GB: The biology of ovarian cancer: new opportunities for translation. Nat Rev Cancer 2009, 9:415-428.

4. Prossnitz ER, Arterburn JB, Smith HO, Oprea TI, Sklar LA, Hathaway HJ: Estrogen signaling through the transmembrane $\mathrm{G}$ protein-coupled receptor GPR30. Annu Rev Physiol 2008, 70:165-190.

5. Filardo EJ, Quinn JA, Frackelton AR Jr, Bland KI: Estrogen action via the G protein-coupled receptor, GPR30: stimulation of adenylyl cyclase and CAMP-mediated attenuation of the epidermal growth factor receptor-to-MAPK signaling axis. Mol Endocrinol 2002, 16:70-84.

6. Filardo EJ: Epidermal growth factor receptor (EGFR) transactivation by estrogen via the G-protein-coupled receptor, GPR30: a novel signaling pathway with potential significance for breast cancer. J Steroid Biochem Mol Biol 2002, 80:231-238.

7. Ahola TM, Manninen T, Alkio N, Ylikomi T: G protein-coupled receptor 30 is critical for a progestin-induced growth inhibition in MCF-7 breast cancer cells. Endocrinology 2002, 143:3376-3384.

8. Ahola TM, Alkio N, Manninen T, Ylikomi T: Progestin and G proteincoupled receptor 30 inhibit mitogen-activated protein kinase activity in MCF-7 breast cancer cells. Endocrinology 2002, 143:4620-4626.

9. Ariazi EA, Brailoiu E, Yerrum S, Shupp HA, Slifker MJ, Cunliffe HE, Black MA, Donato AL, Arterburn JB, Oprea TI, Prossnitz ER, Dun NJ, Jordan VC: The G protein-coupled receptor GPR30 inhibits proliferation of estrogen receptor-positive breast cancer cells. Cancer Res 2010, 70:1184-1194.

10. Chan QK, Lam HM, Ng CF, Lee AY, Chan ES, Ng HK, Ho SM, Lau KM: Activation of GPR30 inhibits the growth of prostate cancer cells through sustained activation of Erk1/2, c-jun/c-fos-dependent upregulation of p21, and induction of G(2) cell-cycle arrest. Cell Death Differ 2010, 17:1511-1523

11. Gao F, Ma X, Ostmann AB, Das SK: GPR30 activation opposes estrogendependent uterine growth via inhibition of stromal ERK1/2 and estrogen receptor alpha (ERalpha) phosphorylation signals. Endocrinology 2011, 152:1434-1447.

12. Ignatov $T$, Weissenborn $C$, Poehlmann A, Lemke A, Semczuk A, Roessner A, Costa SD, Kalinski T, Ignatov A: GPER-1 Expression Decreases During Breast Cancer Tumorigenesis. Cancer Invest 2013, 31:309-315.

13. Smith HO, Arias-Pulido H, Kuo DY, Howard T, Qualls CR, Lee SJ, Verschraegen CF, Hathaway HJ, Joste NE, Prossnitz ER: GPR30 predicts poor survival for ovarian cancer. Gynecol Oncol 2009, 114:465-471.

14. Kolkova Z, Casslen V, Henic E, Ahmadi S, Ehinger A, Jirstrom K, Casslen B: The G protein-coupled estrogen receptor 1 (GPER/GPR30) does not predict survival in patients with ovarian cancer. J Ovarian Res 2012, 5:9.

15. Fujiwara S, Terai Y, Kawaguchi H, Takai M, Yoo S, Tanaka Y, Tanaka T, Tsunetoh S, Sasaki H, Kanemura M, Tanabe A, Yamashita Y, Ohmichi M: GPR30 regulates the EGFR-Akt cascade and predicts lower survival in patients with ovarian cancer. J Ovarian Res 2012, 5:35.

16. Wang C, LV X, Jiang C, Davis JS: The putative G-protein coupled estrogen receptor agonist $\mathrm{G}-1$ suppresses proliferation of ovarian and breast cancer cells in a GPER-independent manner. Am J Trans/ Res 2012, 4:390-402.

17. Hayward JL, Carbone PP, Heusen JC, Kumaoka S, Segaloff A, Rubens RD: Assessment of response to therapy in advanced breast cancer. $\mathrm{Br} J$ Cancer 1977, 35:292-298.

18. Ignatov $A$, Ignatov $T$, Weissenborn $C$, Eggemann $H$, Bischoff J, Semczuk A, Roessner A, Costa SD, Kalinski T: G-protein-coupled estrogen receptor GPR30 and tamoxifen resistance in breast cancer. Breast Cancer Res Treat 2011, 128:457-466.

19. Ignatov T, Eggemann H, Semczuk A, Smith B, Bischoff J, Roessner A, Costa SD, Kalinski T, Ignatov A: Role of GPR30 in endometrial pathology after tamoxifen for breast cancer. Am J Obstet Gynecol 2010, 203:595-16.

20. Ignatov A, Robert J, Gregory-Evans C, Schaller HC: RANTES stimulates Ca2+ mobilization and inositol trisphosphate (IP3) formation in cells transfected with G protein-coupled receptor 75. Br J Pharmacol 2006, 149:490-497.

21. Dennis MK, Burai R, Ramesh C, Petrie WK, Alcon SN, Nayak TK, Bologa CG, Leitao A, Brailoiu E, Deliu E, Dun NJ, Sklar LA, Hathaway HJ, Arterburn JB, Oprea TI, Prossnitz ER: In vivo effects of a GPR30 antagonist. Nat Chem Biol 2009, 5:421-427.

22. Ignatov A, Ignatov T, Roessner A, Costa SD, Kalinski T: Role of GPR30 in the mechanisms of tamoxifen resistance in breast cancer MCF-7 cells. Breast Cancer Res Treat 2010, 123:87-96.

23. Filardo EJ, Quinn JA, Bland Kl, Frackelton AR Jr: Estrogen-induced activation of Erk-1 and Erk-2 requires the $\mathrm{G}$ protein-coupled receptor homolog, GPR30, and occurs via trans-activation of the epidermal growth factor receptor through release of HB-EGF. Mol Endocrinol 2000, 14:1649-1660.

24. Arias-Pulido H, Royce M, Gong Y, Joste N, Lomo L, Lee SJ, Chaher N, Verschraegen C, Lara J, Prossnitz ER, Cristofanilli M: GPR30 and estrogen receptor expression: new insights into hormone dependence of inflammatory breast cancer. Breast Cancer Res Treat 2010, 123:51-58.

25. Albanito L, Madeo A, Lappano R, Vivacqua A, Rago V, Carpino A, Oprea TI, Prossnitz ER, Musti AM, Ando S, Maggiolini M: G protein-coupled receptor 30 (GPR30) mediates gene expression changes and growth response to 17beta-estradiol and selective GPR30 ligand G-1 in ovarian cancer cells. Cancer Res 2007, 67:1859-1866.

26. Vivacqua A, Romeo E, De Marco P, De Francesco EM, Abonante S, Maggiolini M: GPER mediates the Egr-1 expression induced by 17 betaestradiol and 4-hydroxitamoxifen in breast and endometrial cancer cells. Breast Cancer Res Treat 2012, 133:1025-1035.

27. Holm A, Baldetorp B, Olde B, Leeb-Lundberg LM, Nilsson BO: The GPER1 Agonist G-1 Attenuates Endothelial Cell Proliferation by Inhibiting DNA Synthesis and Accumulating Cells in the $S$ and G2 Phases of the Cell Cycle. J Vasc Res 2011, 48:327-335.

28. Choi HJ, Fukui M, Zhu BT: Role of cyclin B1/Cdc2 up-regulation in the development of mitotic prometaphase arrest in human breast cancer cells treated with nocodazole. PLoS One 2011, 6:24312.

29. Hans F, Dimitrov S: Histone $\mathrm{H} 3$ phosphorylation and cell division. Oncogene 2001, 20:3021-3027.

30. Toh WH, Nam SY, Sabapathy K: An essential role for p73 in regulating mitotic cell death. Cell Death Differ 2010, 17:787-800.

31. Henic E, Noskova V, Hoyer-Hansen G, Hansson S, Casslen B: Estradiol attenuates EGF-induced rapid UPAR mobilization and cell migration via the G-protein-coupled receptor 30 in ovarian cancer cells. Int J Gynecol Cancer 2009, 19:214-222.

32. Sun W, Yang J: Functional mechanisms for human tumor suppressors. J. Cancer 2010, 1:136-140.

33. Liu Q, Li JG, Zheng XY, Jin F, Dong HT: Expression of CD133, PAX2, ESA, and GPR30 in invasive ductal breast carcinomas. Chin Med J (Engl) 2009, 122:2763-2769.

34. Poola I, Abraham J, Liu A, Marshalleck JJ, Dewitty RL: The Cell Surface Estrogen Receptor, G Protein- Coupled Receptor 30 (GPR30), is Markedly Down Regulated During Breast Tumorigenesis. Breast Cancer (Auckl.) 2008, 1:65-78.

doi:10.1186/1757-2215-6-51

Cite this article as: Ignatov et al:: GPER-1 acts as a tumor suppressor in ovarian cancer. Journal of Ovarian Research 2013 6:51. 